\title{
An adaptive controller for motion control of nanorobots inside human blood vessels
}

\author{
Amir Farahani*1 and Ali Farahani ${ }^{2}$ \\ ${ }^{1}$ Ashtian Branch, Islamic Azad University, Ashtian, Markazi, Iran \\ ${ }^{2}$ Kharazmi University, Tehran, Iran
}

\begin{abstract}
The rise of nanorobots for medical diagnostics and treatments is arriving in the near future. In this study, a control method is designed so that the motion of a nanorobot can be controlled inside the blood vessel of the human body when we use them for medical therapies. A kinematic model of nanorobot in MATLAB/SIMULINK is used for demonstrating the movement of nanorobots to examine the performance of proposed control mechanism. The nanorobot system used in this study is a nanorobot with very small dimensions $(0.5 \mu \mathrm{m})$ with a rigid body. It was assumed that the damaged part of the vessel wall is detected by the nanorobot with the help of the signals transmitted from the part. We applied an adaptive control algorithm and with this type of controller, the nanorobot motion was optimized. Our results showed that by using the proposed method, the system was controlled such that the nanorobot moved towards the damaged area and reached the target destination with an improved accuracy. Simulations indicated the efficiency of this control method with respect to the nonlinear velocity equation of the nanorobot.
\end{abstract}

KEY WORDS: MEDICAL THERAPY, NANOROBOTS, NAVIGATION, OPTIMIZATION, CONTROL SYSTEM, ADAPTIVE METHOD

\section{INTRODUCTION}

Platelets are plasma elements which are spherical or oval shaped with 2-5 microns in diameter (Warkentin, 2009). Blood platelets are blood cells which exist in our blood. When they recognize a part of the blood vessel is damaged, they intertwine to stop the bleeding. In other words, when there is a wound in the body, the platelets stick together, and create a blood clot; as result, it cause a hemostatic process. Platelets also are called thrombocytes because a clot also is called a thrombus. Thrombocytopenia is a condition in which the platelets count is

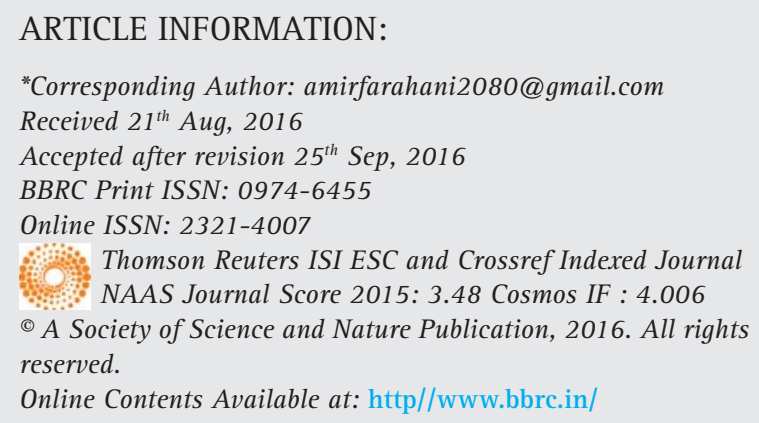


less than 150,000 per microliter which causes excessive bleeding. In this case, the patient may enter the shock from blood loss. Or it may occur inside the brain causing intracranial hemorrhage which causes death or paralysis of the patient. There are many ways to treat thrombocytopenia. One common method is the Platelet transfusions. Normal platelet count in an adult is 150000$400000 / \mu \mathrm{L}$. After the transfusion, platelets remain only about ten days, and then, they are destroyed. Thus, the patient needs the transfusion of platelets about two to three times per month. One of recent techniques that is used to heal the wounds is the use of nanorobots which within the human body, acts like artificial platelets. They are expected to enable diagnosis, medical therapies, and surgery (Montemagno and Bachand, 1999; Freitas 1998, 1999 and 2005; Cavalcanti, 2003).

Nanorobots can travel inside the body. They can detect different patterns of chemicals in the body, monitor their biochemical and biomechanical characteristics and store the observations based on an internal clock in their memories (Hogg and Kuekes, 2006). With the help of nanomedicine diseases can be easily cured with no side effects and no surgery is required (Yadav et al. 2011).There are many studies that have investigated the use of nanorobots for medical treatment (Abeer , 2012; Kharwade et al. 2013;Manjunath and Kishore, 2014).

Most researches on nanorobots have been done for different type of automated and accurate medical target identification (Cavalcanti et al. 2008). Nanorobots are increasingly important in today's world, but there is still no good framework to design these kinds of robots, although in this field recently some studies have been carried out (e.g. Behkam and Sitti, 2006; Arora and Saini, 2013 and Farr et al. 2014).

Cavalcanti et al. (2009) presented a method to release nanorobots inside the blood vessels to identify aneurysm in the brain. Al-Arif et al. (2011) investigated the possibility of using Nanorobots in finding damaged nerve and delivering medicine to cure the demyelination. Due to the small size of these robots, the designed robots are very complicated and consist of very small components. Boonrong and Kaewkamnerdpong (2011) proposed a swarm intelligence based control mechanism for swarm nanorobots that operate as artificial platelets to search for wounds. For both industrial and medical applications such as microsurgery, Pan et al. (2011) developed a prototype with a rotatable head and a body that has legs and fins. Douglas et al. (2012) described a DNA nanobot shaped like a hexagonal tube, with its two halves connected by a latched hinge. When the little device recognizes a target cell based on its surface proteins, the two halves swing open like a clam to deliver a tiny but deadly cargo of drugs or nanoparticles.
Jaiswal et al. (2013) studied the use of an artificial nano-medical erythrocyte which duplicates all of the important functions of the red blood cell by providing treatment for anaemia, heart attack, choking, lung diseases, asphyxia, and other respiratory problems. These nano-robots are able to keep a patient's tissues safely oxygenated for up to about 4 hours (at maximum dosage) if their heart has stopped beating in case of a heart attack.

In this study, a method is proposed to control the nanorobot motion inside the human blood vessel by performing an adaptive control algorithm. The importance of the nanorobot motion control within the vessel is revealed when the nanorobot wants to move towards the wall to detect and treat the wounds and inflammation of the blood vessel wall. The structure of this paper is as follows: first we introduce the kinematic model of our nanorobot system, and present its governing equations; next, nanorobot control system is designed and its control algorithm is provided; next section reports the results of the simulation to evaluate the performance of the system; and in the final section we present our conclusions according to the results of stimulation

\section{MATERIAL AND METHODS}

\section{NANOROBOT SYSTEM}

In this paper we tried to optimize the movement of a nanorobot inside human blood vessel. The nanorobot system used in this study is a nanorobot with very small dimensions $(0.5 \mu \mathrm{m})$ with a rigid body. Figure 1 shows the kinematic model of this system.

In this figure, $\phi$ is the rotational angle of the tip of the nanorobot, $\theta$ is the angel between the body axis and the axis $\mathrm{X}, \mathrm{l}$ is the nanorobot length, $k$ denotes the curvature of the reference trajectory, and $O$ is the center of rotation. Considering the rigid body of nanorobots, we will have:

$$
\begin{aligned}
& \mathrm{x}_{\mathrm{r}}=\mathrm{x}_{\mathrm{f}}-\mathrm{l} \cos \theta \\
& \mathrm{y}_{\mathrm{r}}=\mathrm{y}_{\mathrm{f}}-1 \sin \theta
\end{aligned}
$$

By deriving from equation (1) and (2), we will have:

$$
\begin{aligned}
& \dot{\mathrm{x}}_{\mathrm{r}}=\dot{\mathrm{x}}_{\mathrm{f}}+\dot{\theta} \mathrm{l} \sin \theta \\
& \dot{\mathrm{y}}_{\mathrm{r}}=\dot{\mathrm{y}}_{\mathrm{f}}-\dot{\theta} \mathrm{l} \cos \theta
\end{aligned}
$$

According to figure 1, we have:

$$
\dot{\mathrm{x}}_{\mathrm{f}}=\mathrm{v} \cdot \cos (\theta+\phi)
$$




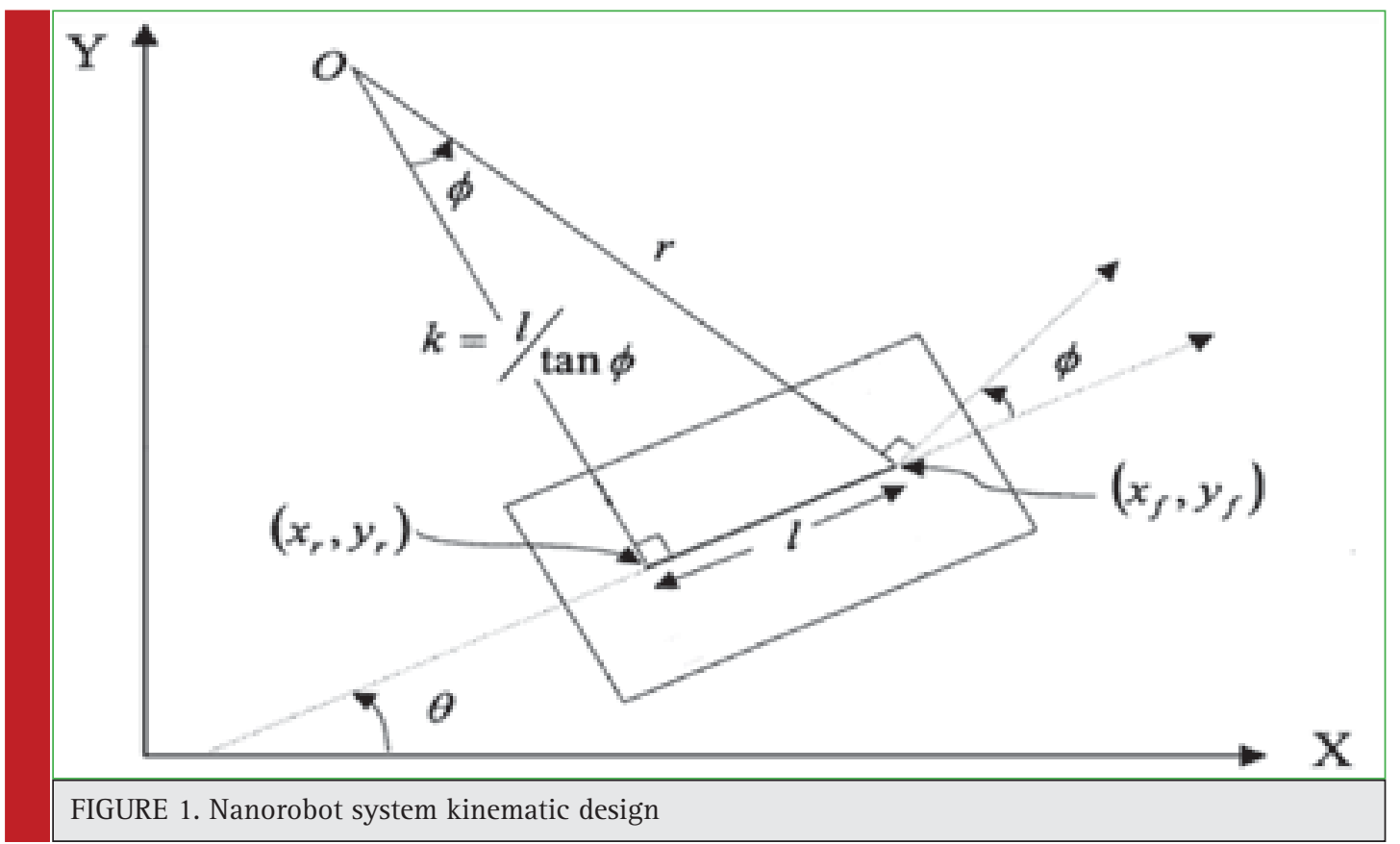

$$
\begin{aligned}
& \dot{\mathrm{y}}_{\mathrm{f}}=\mathrm{v} \cdot \sin (\theta+\phi) \\
& \dot{\theta}=\mathrm{v} \cdot \frac{\sin \phi}{\mathrm{l}}
\end{aligned}
$$

Equations (5), (6) and (7) express nanorobots kinematic equations. These equations are used to calculate the next state of the robot moving forward to the destination which is the vessel wall.

In this study, it is assumed that the damaged part of the vessel wall is detected by the nanorobot with the help of the signals transmitted from the part. The path to the damaged part is determined as a fifth-order equation which is presented as a reference trajectory in equation (8).

$$
y(x)=a_{0}+a_{1} x+a_{2} x^{2}+a_{3} x^{3}+a_{4} x^{4}+a_{5} x^{5}
$$

The reference path is shown in figure 2 .

To find the coefficients of the equation for the reference trajectory, the following boundary conditions are used:

$$
\begin{array}{rr}
\mathrm{y}\left(\mathrm{x}_{\mathrm{s}}\right)=\mathrm{y}_{\mathrm{s}} & \mathrm{y}\left(\mathrm{x}_{\mathrm{g}}\right)=\mathrm{y}_{\mathrm{g}} \\
\dot{\mathrm{y}}\left(\mathrm{x}_{\mathrm{s}}\right)=0 & \dot{\mathrm{y}}\left(\mathrm{x}_{\mathrm{g}}\right)=0
\end{array}
$$

Where, $\mathrm{X}_{\mathrm{s}}=$ horizontal component of the initial point, $\mathrm{Y}_{\mathrm{s}}$ = vertical component of the initial point, $\mathrm{X}_{\mathrm{g}}=$ horizontal component of the terminal point, and $Y_{g}=$ vertical component of the terminal point. According to the reference trajectory, the nanorobot follows the path. To achieve this goal, we designed a controller that with determining the angle of $\varphi$, it be able to control the angle $\theta$ in such a way that the nanorobot motion can move according to the reference trajectory. With this algorithm, nanorobot can reach the affected area with high accuracy after traveling in the motion path, arrive the damaged part. System error is calculated using equations (10) and (11) which are appropriate criteria for evaluating the system performance. Equation (10) computes the error of the horizontal component of nanorobot motion relative to the horizontal component of the reference trajectory:

$$
\mathrm{e}_{\mathrm{y}}(\mathrm{k})=\mathrm{x}_{\text {trajectory }}(\mathrm{k})-\mathrm{x}_{\text {motiion }}(\mathrm{k})
$$

While, equation (11) calculates the error of the vertical component of nanorobot motion relative to the vertical component of the reference trajectory.

$$
\mathrm{e}_{\mathrm{y}}(\mathrm{k})=\mathrm{y}_{\text {trajectory }}(\mathrm{k})-\mathrm{y}_{\text {motion }}(\mathrm{k})
$$

\section{DESIGN OF THE CONTROLLER}

The controller designed for this purpose is an adaptive controller. The basis for using adaptive controller is to control the system with the presence of uncertainty. Although uncertainty means the presence of the anonymity of parameters and system dynamics, but an adaptive controller generally requires parameters estimation to limit the system output error. In other words, the expectation of an adaptive controller is not the estimation of system's unknown parameter but controller parameter estimation in a way that the error remains 


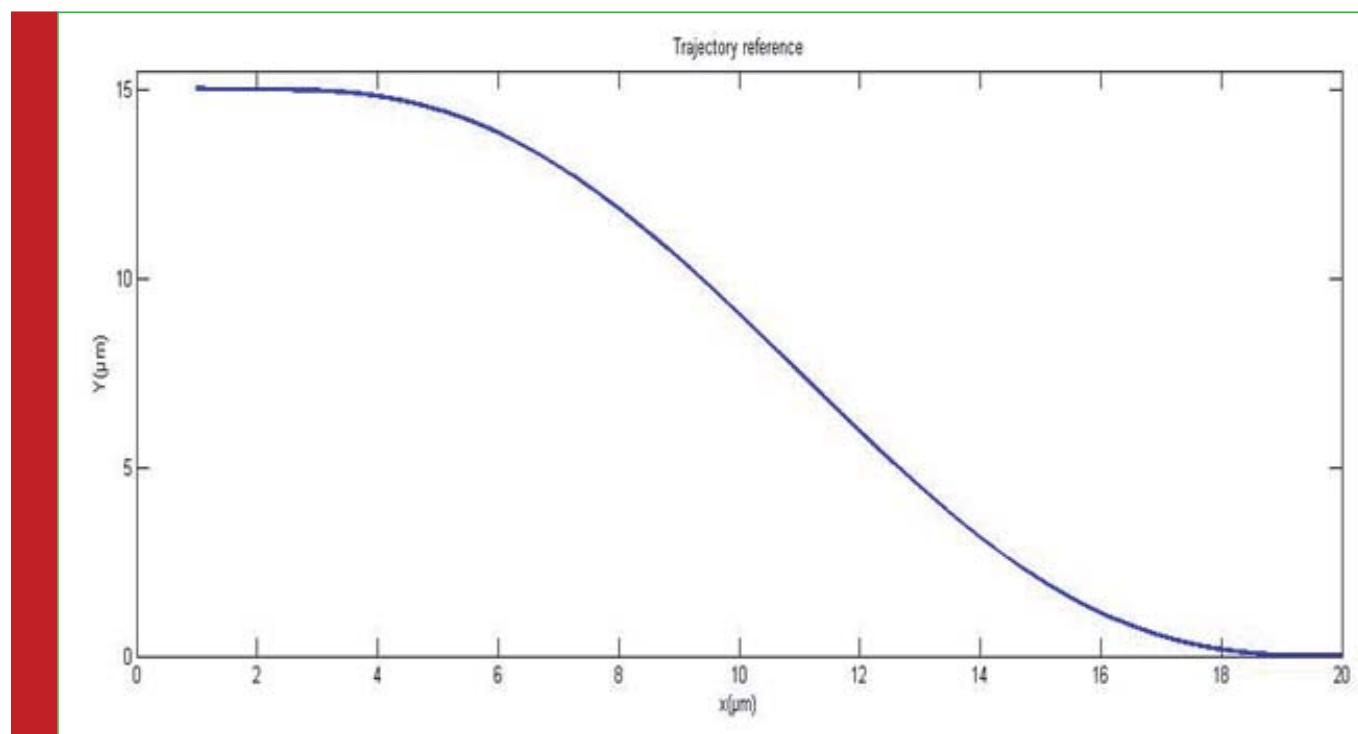

FIGURE 2. Reference trajectory of the nanorobot system

limited and acceptable. However, in case of persistent irritation in the system, we can estimate the parameters of the system.

The adaptive control of non-linear systems generally has no same rules and procedures. However, in this system, we can find out that because of the structure of the state space equations, the third equation of the nanorobot system's kinematic equations can be used. In other words, by the state control of in equation (12), $\mathrm{x}$ and $\mathrm{y}$ can be controlled in equations (13) and (14):

$$
\begin{gathered}
\dot{\theta}=v \cdot \frac{\sin \phi}{L} \\
\phi=\arcsin \left(\left(\frac{\mathrm{L}}{\mathrm{v}}\right)\left(\dot{\theta}_{\mathrm{m}}-\lambda\left(\theta-\theta_{\mathrm{m}}\right)\right)\right)
\end{gathered}
$$

Where, $\mathrm{L}=$ nanorobot length, $\mathrm{v}=$ nanorobot velocity, $\dot{\theta}=$ derived reference angle between the body axis and the axis $\mathrm{x}, \lambda=$ controller parameter, and $\theta_{\mathrm{m}}=$ the reference angle between the body axis and the axis $\mathrm{x}$. Now, by having all the values, the system tracking error will tend to zero with the appropriate choice of $\lambda$. As it was mentioned before, the motion mechanism of nanorobot is such that the blood flow in vessels is the only driving force or thrust. The blood velocity in the vessel is obtained as:

$$
\mathrm{V}=\frac{\mathrm{a}^{2}}{4 \mu} \frac{\mathrm{dP}}{\mathrm{dx}}\left[\left(\frac{\mathrm{y}}{\mathrm{a}}\right)^{2}-\frac{\mathrm{y}}{\mathrm{a}}\right]
$$

Where $\frac{\mathrm{dP}}{\mathrm{dx}}=$ pressure gradient, $\mu=$ viscosity coefficient, $=$ vessel diameter, and $=$ longitudinal location of nanorobot. We assumed that nanorobot is located between the end of the arterial and venous vessels.
According to Sembulingam and Sembulingam (2012), pressure gradient in this area is $15 \mathrm{~mm} \mathrm{hg}$. Also, according to Hogg and Kuekes (2006), the blood viscosity coefficient is 0.04 . The vessel diameter varies in different parts of the body.In this study, the vessel diameter was considered as $30 \mu \mathrm{m}$ and nanorobot length as $0.5 \mu \mathrm{m}$. Therefore, considering the provided values for the parameters, the blood velocity which is equal to the velocity of nanorobot motion can be obtained. In equation (14), with smaller $\mathrm{y}$, we will have less fluid velocity. In other words, when nanorobot gets closer to the vessel wall, its movement speed is reduced. The velocity of blood has the highest value in the center of the vessel, and with higher distance from the center, its velocity is decreased.

We assumed that nanorobot is in motion with the highest speed in the center of vessel, and by recognizing the damaged blood vessel wall, it begins to move from the center to the wall. By substituting equation (12) into equation (11), the is controlled, and by substituting the controlled into the motion equations of (5) to (7), Nanorobot path can be controlled well with respect to the reference path. Figure 3 illustrates the overall block diagram of the system with the controller.

\section{RESULTS AND DISCUSSION}

In this study, MATLAB/ SIMULINK software was used to simulate Nanorobot motion within the blood vessel. By using equation (5) to (7), nanorobot motion from the center of the vessel to the damaged part was plotted as shown in figure 4.

By comparing the reference trajectory path with nanorobot motion path (Fig.5), we can see that the 


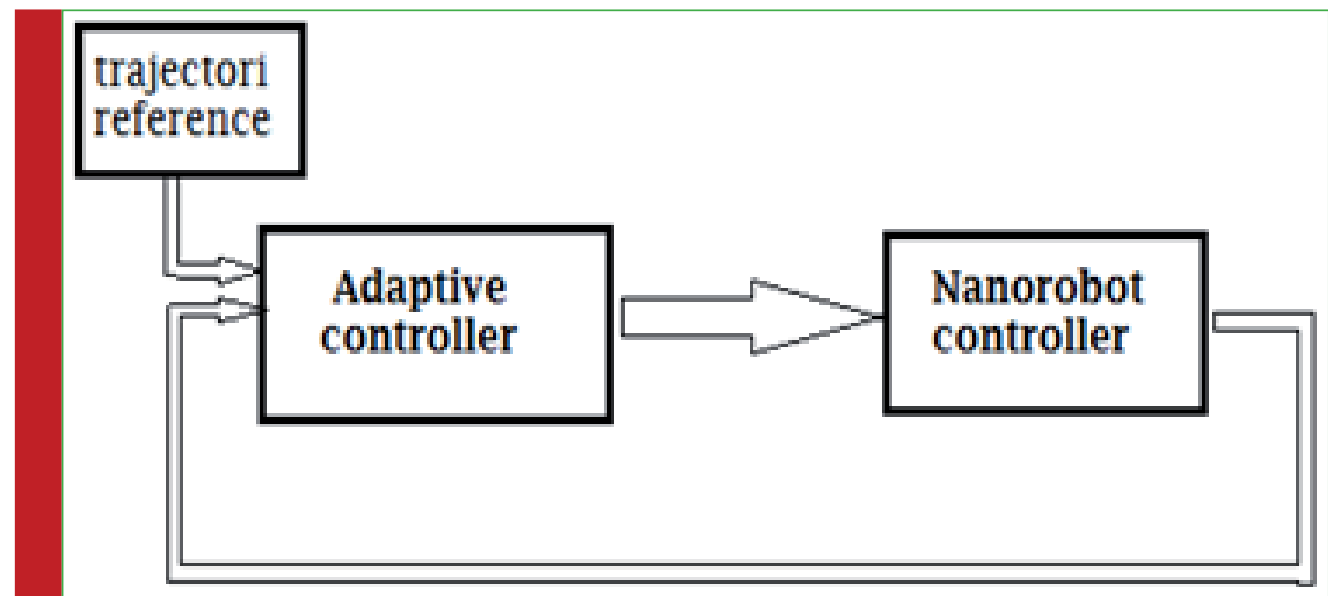

FIGURE 3. Block diagram of the proposed system

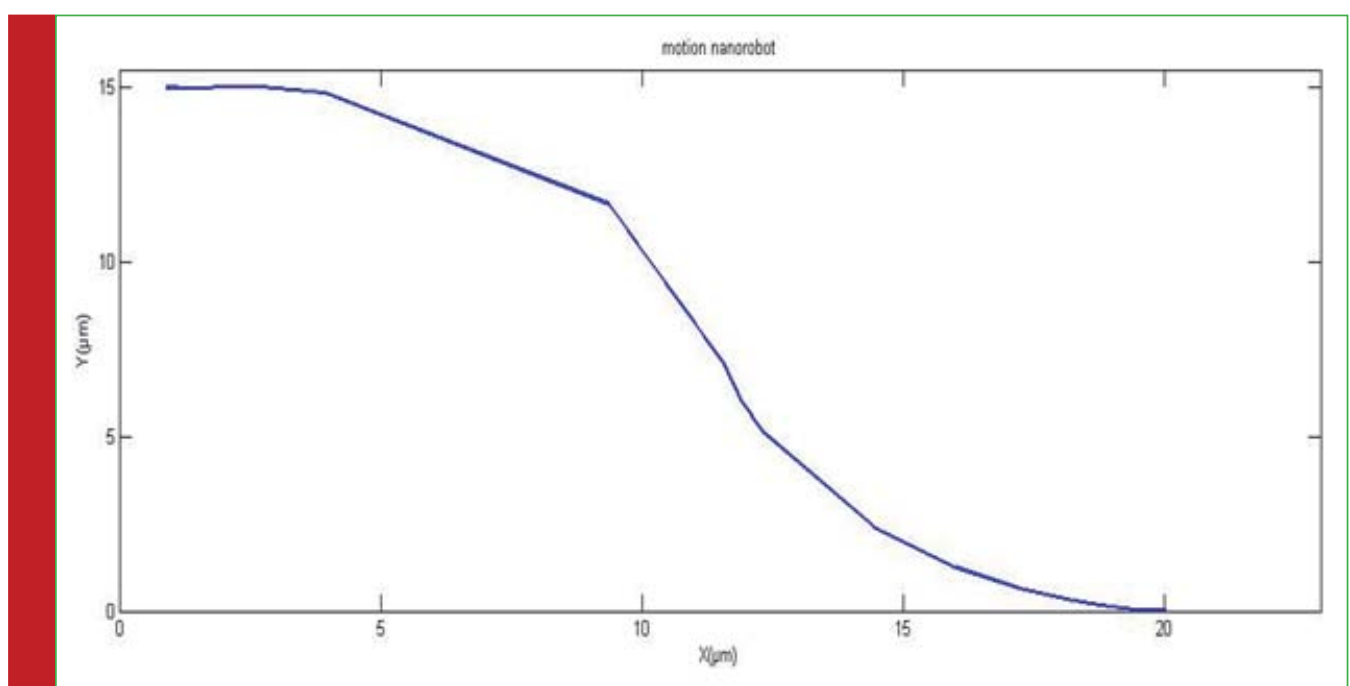

FIGURE 4. Nanorobot motion

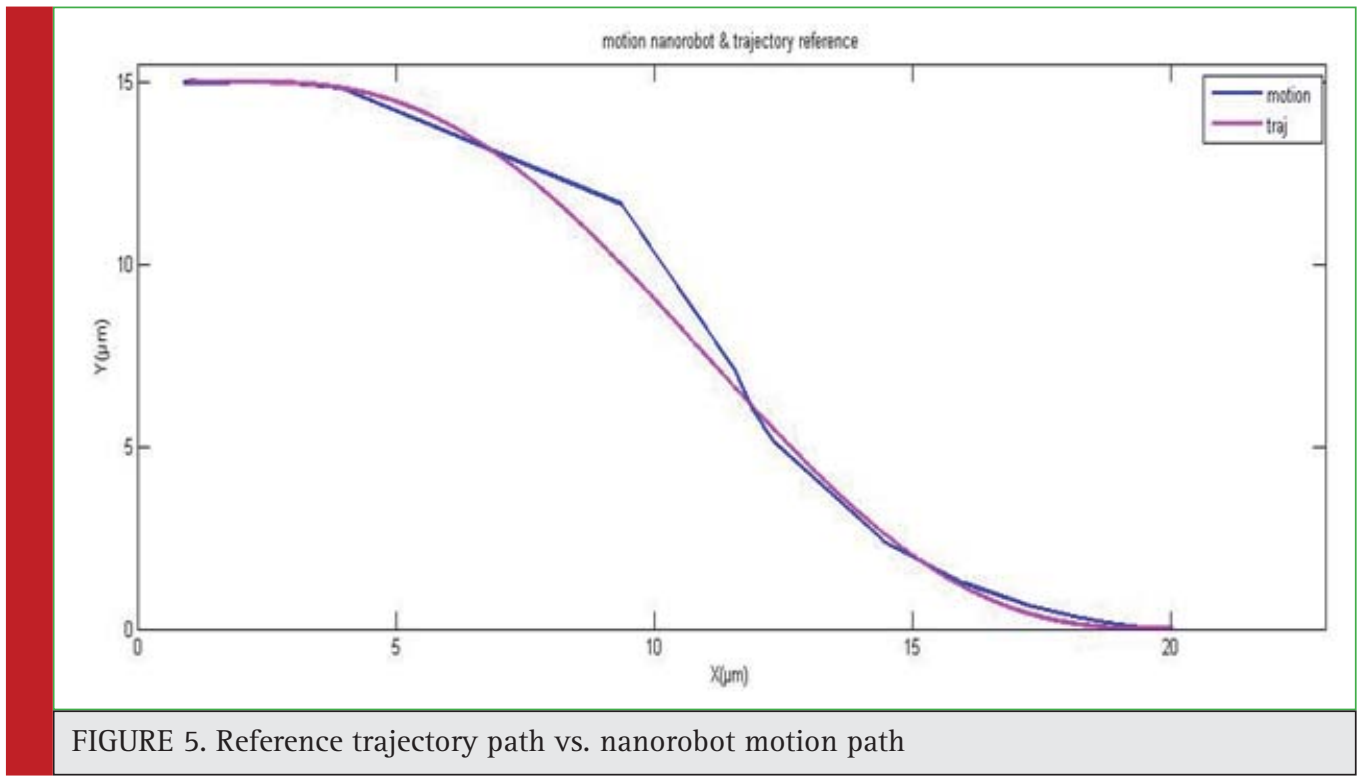




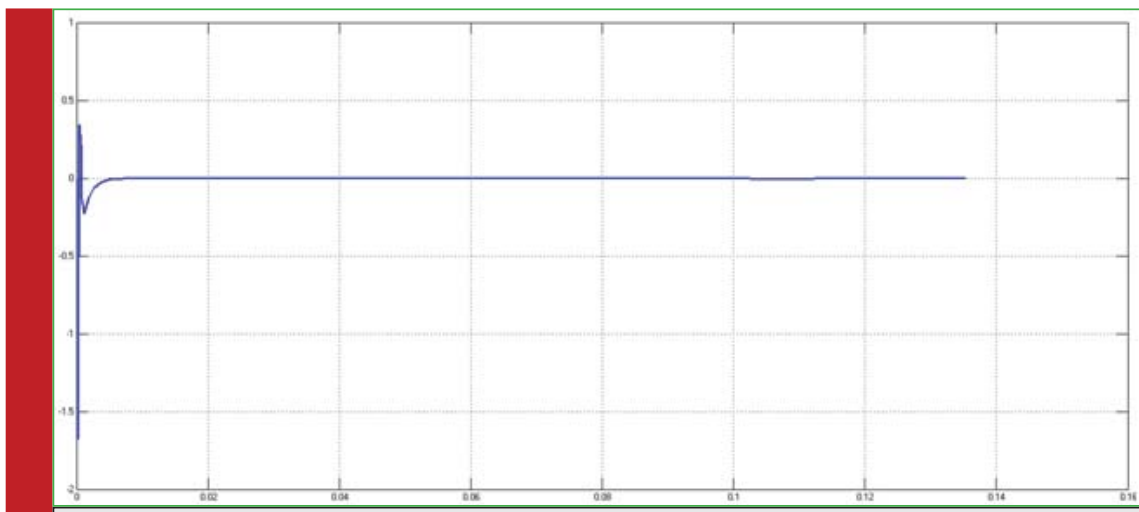

FIGURE 6. Motion error of nanorobot in vertical direction

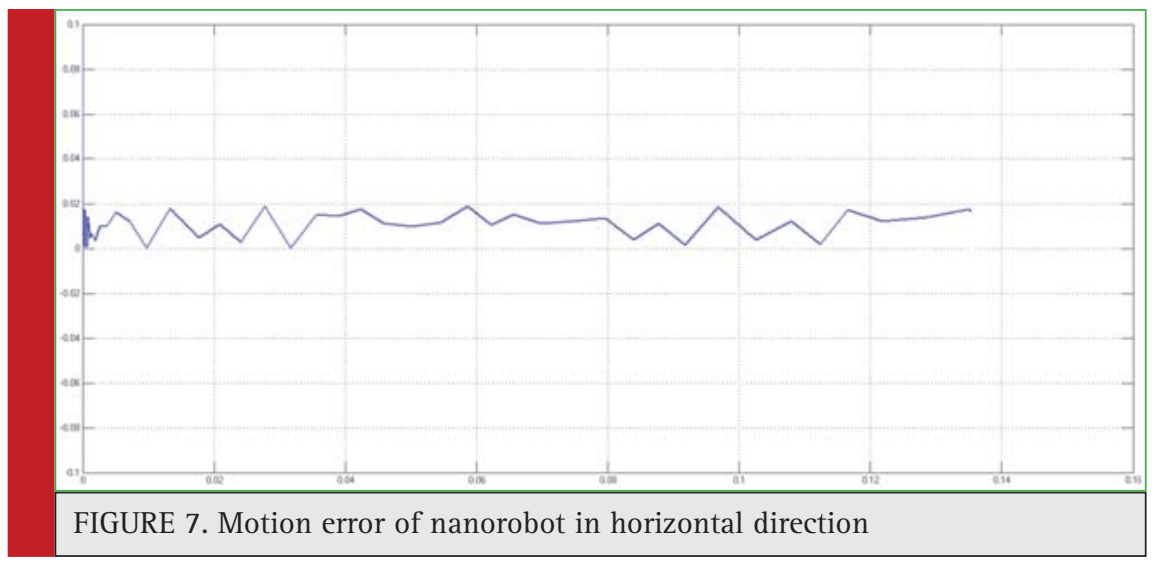

motion path curve of the nanorobot is largely matches the trajectory path curve, and there have little difference with each other. This ensures the nanorobots to reach the desired destination.

Motion error graph of nanorobot in two vertical and horizontal directions are shown in Figure 6 and 7, respectively. In vertical direction, it can be clearly seen that most error rate at the beginning of the movement and the time it starts, the amount of which is equal to
$1.6 \mu \mathrm{m}$ which is negligible. After 0.001 seconds, its error rate is drastically reduced and approaches to zero (Fig. 6). In horizontal direction, the greatest amount of error is $0.02 \mu \mathrm{m}$ which is negligible (Fig. 7). Given the results of simulation, it can be well observed that the proposed controller can properly navigate the nanorobot, and by using this controller, we can control the motion off nanorobot to the location of the damaged vessel wall by controlling its movement angle.

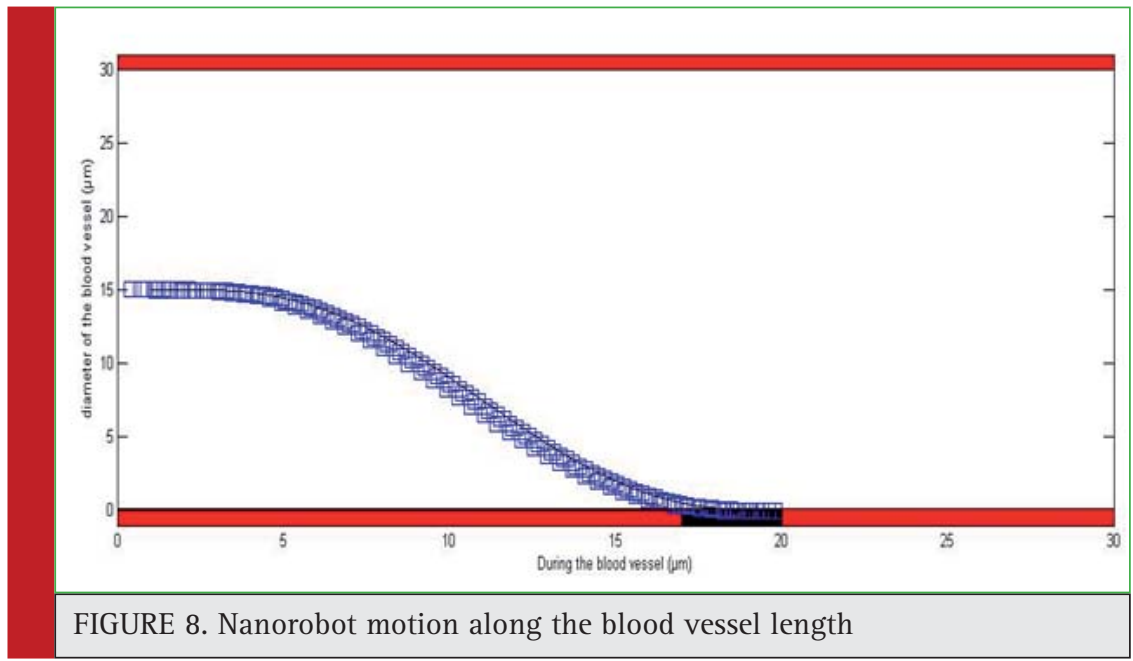


Nanorobot motion within the vessel is shown in figure 8. The red section represents the blood vessel wall; the blue lines show the nanorobot motion path, and the black lines are the damaged parts on the blood vessel wall. We can see that the nanorobot moves from the center of the vessel, and after moving in a given direction provided by the controller, it reaches the damaged part.

\section{CONCLUSION}

In this study we attempted to design an adaptive controller to optimize the motion of nanorobots within the within the blood vessel. The results of the simulation showed that the proposed control method, by identifying the functional characterization of nano-robots such as transport capability, biocompatibility, planning, receiving signals and its generation, had good performance in controlling the nanorobot motion, and its efficiency is high. The experimental results and simulations which were based on considering the blood vessel as a rigid pipe, showed that with the help of the adaptive controller, nanorobot followed the determined reference trajectory path by the signal received from the damaged area, and was moved to the target destination with an error of about $0.01 \mu \mathrm{m}$. The design of this controller makes it possible for us to be able to use nanorobots for e.g. venous injury treatment, cancer treatment, treatment of damaged cells, or laparoscopy with great accuracy. We suggest that a study to be conducted by considering the blood vessel as a flexible tube to examine nanorobot motion inside the vessel and the relationship among several nanorobots in the body to enhance their efficiency.

\section{REFERENCES}

Abeer, S. (2012). Future Medicine: Nanomedicine. Journal of International Medical Science Academy, Vol. 25, No. 3: Pages 187-192.

Al-Arif, S.M.M.R. M., Quader, N., Shaon, A. M., Islam, K. K.(2011). Sensor based autonomous medical nanorobots. A cure to demyelination. Journal of Selected Areas in Nanotechnology Vol. 2, No. 11: Pages 1-7.

Arora, R. K., Saini, R. P.(2013). Biosensors: Way of Diagnosis. International Journal of Pharmaceutical Sciences and Research Vol. 4, No.7: Pages 2517-2527.

Behkam B., Sitti, M. (2006).Towards Hybrid Swimming Microrobots: Bacteria Assisted Propulsion of Polystyrene Beads. In: Proceedings of the 28th IEEE EMBS Annual International Conference, New York City, USA, Pages 2421-2424.

Boonrong,P.and Kaewkamnerdpong, B.(2011). Canonical PSO based nanorobot control for blood vessel repair. World Academy of Science, Engineering and Technology Vol. 58: Pages 511-516.
Cavalcanti A. (2003). Assembly automation with evolutionary nanorobots and sensor-based control applied to nanomedicine. IEEE Transactions on Nanotechnology Vol. 2, No. 2: Pages 28-27.

Cavalcanti, A., Shirinzadeh B., Freitas Jr R.A., and Hogg, T. (2008). Nanorobot architecture for medical target identification. Nanotechnology Vol. 19, No. 1: Pages 1-15.

Cavalcanti, A., Shirinzadeh, B., Fukuda, T., Ikeda, S. (2009). Nanorobot for brain aneurysm. International Journal of Robotics Research archive Vol. 28: Pages 558-570.

Douglas, S.M., Bachelet, I., Church, G.M. (2012). A Logic-Gated Nanorobot for Targeted Transport of Molecular Payloads. Science, Vol. 335, No. 6070: Pages 831-834.

Farr, R., Choi, D.S., Lee, S.W. (2014). Phage-Based Nanomaterials for Biomedical Applications. Acta Biomaterialia Vol. 10, No. 4: Pages 1741-50.

Freitas R. A. Jr. (1998). Exploratory design in medical nanotechnology: A mechanical artificial red cell. Artificial Cells, Blood Substitutes, and Immobilization Biotechnology Vol. 26: Pages 411-430.

Freitas R. A. Jr (1999). Nanomedicine vol I: Basic Capabilities. Landes Bioscience: Georgetown, TX.

Freitas R. A. Jr. (2005). Nanotechnology, nanomedicine and nanosurgery. International Journal of Surgery Vol. 3: Pages 1-4.

Jaiswal, A., Thakar, H., Atanukumar, B., Krunali, T., and Meshram, D. B. (2013). Nanotechnology revolution: respirocytes and its application in life sciences. Innovative Journal of Life Sciences, Vol. 1, No.1: Pages 8-13, 2013.

Hogg, T., Kuekes, P.J. (2006) . Mobile Microscopic Sensors for High Resolution in Vivo Diagnostics. Nanomedicine: Nanotechnology, Biology, and Medicine Vol. 2, No. 4: Pages 239-247.

Kharwade, M., Nijhawan, M., and Modani S. (2013). Nanorobots: A Future Medical Device in Diagnosis and Treatment. Research Journal of Pharmaceutical, Biological and Chemical Sciences, Vol. 4, No. 2: Pages 1299-1307.

Manjunath, A., and Kishore, V. (2014). The Promising Future in Medicine: Nanorobots. Biomedical Science and Engineering, Vol. 2, No. 2: Pages 42-47.

Montemagno C. D., Bachand G. D. (1999). Constructing nano mechanical devices powered by biomolecular motors. Nanotechnology Vol. 10: Pages 225-231.

Pan, Q., Guo, S., Okada, T.(2011). A novel hybrid wireless Microrobot. International Journal of Mechatronics and Automation Vol. 1, No. 1: Pages 60-69.

Sembulingam, K., Sembulingam P.(2012). Essentials of Medical Physiology ( $6^{\text {th }}$ Edn.). Jaypee Brothers Medical Publishers Ltd: New Delhi, India.

Warkentin, T. E. (2009). Thrombocytopenia due to platelet destruction and hypersplenism, In: Hematology: Basic Principles and Practice. Pp 2113-2131 (Ed) R. Hoffman. Churchill Livingstone Elsevier: Philadelphia, US.

Yadav, A., Ghune, M., Jain, D.(2011). Nano-medicine based drug delivery system. Journal of Advanced pharmacy Education and Research Vol. 1, No. 4: Pages 201-213. 